\title{
The role of infinity with signs in mathematics
}

\author{
Erkara Aydos Zh. ${ }^{1}$, Raushan Kadyrova S. ${ }^{2}$ \\ ${ }^{1}$ Kazakh National Technical University after K.I.Satpaev, Almaty, Kazakhstan \\ ${ }^{2}$ Kazakh British Technical University, International School of Economics and political Sciences
}

\section{Email address:}

erkaraai@mail.ru(Erkara A. Zh.), raushankadyrova19@gmail.com(Raushan K. S.)

To cite this article:

Erkara Aydos Zh., Raushan Kadyrova S.. The Role of Infinity with Signs in Mathematics. International Journal of Renewable and Sustainable Energy. Vol. 2, No. 3, 2013, pp. 72-76. doi: 10.11648/j.edu.20130203.12

\begin{abstract}
The article shows the significance of signs $+\infty$ and $-\infty$ (infinity with signs) for a full exposition of some of the problematic materials in mathematics. With its help smoothness of the curve, specified by functions with finite and infinite derivatives at the points, is determined. The properties of the function $f$, with which you can set the smoothness of the curve $\Gamma: y=f(x), x \in[a ; b]$, are indicated.
\end{abstract}

Keywords: Limit, Derivative, Smooth Curve, Infinite Derivative, Tangent, Angular Function

In this article we show how to bridge the gaps of some theoretical aspects of mathematics through infinity $+\infty$ and $-\infty$. But first we give some examples of their participation. One of them is the so-called mean-value theorem. For clarity, we consider the Lagrange theorem, whose condition is formulated in the language of infinite derivative.

Theorem $\overline{1}$ (Lagrange). If the function $f(x)$ is continuous on the interval $[a, b]$ and its derivative exists (in the broadest sense) on the interval $(a, b)$, then there is a point $c \in(a, b)$, for which the equality

$$
f(b)-f(a)=(b-a) f^{\prime}(c), a<c<b .
$$

is true.

But in many textbooks the mean-value theorem is formulated in terms of a finite derivative, and therefore cannot be applied to functions with infinite derivatives, i.e. the range of applicability narrows (for example, for the function

$$
f(x)=\sqrt[5]{x-4}, 3 \leq x \leq 5
$$

with an infinite derivative ${ }^{+\infty}$ at a point $x=4$, conclusion of Lagrange's theorem is satisfied).

Another example. It is known that if the function $\mathrm{f}$ has a finite derivative at a point $x_{0}$ and $f^{\prime}\left(x_{0}\right)>0$, , then at this point the function increases.

This statement is powerless, for example, to identify the point of increase $x=0$ of the function $f(x)=\sqrt[3]{x}$, since at this point the function has infinite derivative $f^{\prime}(0)=+\infty$.
This statement can be strengthened by $+\infty$ and $-\infty$ infinities, as follows:

If function $f$ has at the point $x_{0} a$ derivative (in the broadest sense) and

$$
f^{\prime}\left(x_{0}\right)>0\left(f^{\prime}\left(x_{0}\right)>0\right)
$$

then the function increases(decreases) at this point.

Indeed,

$$
f^{\prime}\left(x_{0}\right)=\lim _{x \rightarrow x_{0}} \frac{f(x)-f\left(x_{0}\right)}{x-x_{0}}=+\infty
$$

means that

$$
\begin{gathered}
\forall E>0, \exists \delta\left(x_{0} ; E\right),\left(\forall x: 0<\left|x-x_{0}\right|<\delta\right) \Rightarrow \\
\left.\frac{f(x)-f\left(x_{0}\right)}{x-x_{0}}\right\rangle E>0
\end{gathered}
$$

Therefore, by definition, $x_{0}$ is the point of increase of the function $f$. In the case of $f^{\prime}\left(x_{0}\right)<0$, the proof is analogous. Since for the function $f(x)=\sqrt[3]{x}$ we have $f^{\prime}(0)=+\infty$, then, by the last assertion, the fucntion increases at the point $x=0$.

As a third example, consider the definition of a smooth curve and on its basis, go to the main subject of the article.

Definition 1. The curve $\Gamma \subset R^{3}$, given by parametric equation $x=x(t), y=y(t), z=z(t), t \in[a ; b]$, is called smooth, if $x(t), y(t), z(t)$ - continuous functions with continuous derivatives in the interval $[a ; b]$, which are not simultaneously equal to zero.

Semicircle

$$
\Gamma_{1}: x=\sqrt{1-t^{2}}, y=t,-1 \leq t \leq 1 ;
$$


cubic parabola

$$
\Gamma_{2}: y=\sqrt[3]{t}, x=t,-\infty \leq t \leq+\infty ;
$$

indeed, all types of curves, introduced with the functions

$$
y=\sqrt[2 n+1]{(t-a)^{2 k-1}}, x=t, a-l \leq t \leq a+m
$$

Where

$$
n, k \in \mathbb{N}, k \leq n, n>1, a, l, m \in \mathbb{R}, l>0, m>0,
$$

according to definition 1, are not smooth, because functions, which represent them, are not differentiable in these intervals. The question arises: if the functions, parameterizing the curve, have derivatives, which go to infinity in the considered interval, then the curve can be regarded as a non-smooth? Of course, no, since one and the same curve may have a differentiable parameterization as well as parameterization of non-differentiable functions (see below).

The foregoing is the motivation for the following modification of the definition of a smooth curve (see [1] $\S 6.5$, where the definition is given for more general situation).

Definition 2. The curve $\Gamma \subset R^{3}$ is called smooth on $[a ; b]$ if it can be set, using the equations

$$
\Gamma: x=x(t), y=y(t), z=z(t), t \in[a ; b]
$$

where

$$
x(t), y(t), z(t)-
$$

continuous functions with continuous derivatives on an interval $[a ; b]$, which are not simultaneously equal to zero.

For example, a semicircle $\Gamma_{1}$, discussed above can be parameterized in the form of

$$
x=\cos t, y=\sin t,-\frac{\pi}{2} \leq t \leq \frac{\pi}{2},
$$

that is why semicircle $\Gamma_{1}$ is a smooth curve in the sense of definition 2; a cubic parabola $\Gamma_{2}$ can be represented as

$$
y=\operatorname{sh} t, x=\operatorname{sh}^{3} t,-\infty \leq t \leq+\infty ;
$$

so that the curve $\Gamma_{2}$, according to the definition 2 , is smooth too.

However, the need to search for a parameterization of the curve with functions with suitable properties is lack of definition 2 - the way of the parameterization of the one and same curve, as mentioned above, is not the only one (for example, the semicircle $\Gamma_{1}$ can also be represented as

$$
\left.y=\frac{2 t}{1+t^{2}}, x=\frac{1-t^{2}}{1+t^{2}},-1 \leq t \leq 1, \text { etc. }\right)
$$

generally speaking, whether there is a parameterization with desired properties or not is not clear in advance. Below we will address the problems inherent to the definitions 1 and 2, and will also indicate the characteristic of the function $f$ with which the smoothness of the curve

$$
\Gamma: y=f(x), x \in[a ; b],
$$

can be established.

\section{The Basic Concepts}

Let us complete the set $\mathbb{R}$ with two improper elements ("infinite numbers")

$-\infty$ and $+\infty$, assuming that for any $x \in \mathbb{R}$ there is

$$
-\infty<x<+\infty \text {. }
$$

In the case, when

$$
\lim _{x \rightarrow x_{0}} f(x)=A
$$

where $A \in[a ; b]$, we say, as usual, that the function $f$ at the point $x_{0}$ has a limit A, where if $A \in(-\infty ;+\infty-$ "finite limit". For example, the function $f(x)=\frac{1}{(x-1)^{2}}$ has the limit equal to $+\infty$ at the point $x=1$, and the function $f(x)=\frac{1}{x-1}$ at this point has no limit, as

$$
f(1-)=-\infty \neq+\infty=f(1+) .
$$

Similarly, if we have

$$
f^{\prime}\left(x_{0}\right)=\lim _{x \rightarrow x_{0}} \frac{f(x)-f\left(x_{0}\right)}{x-x_{0}},
$$

Where

$$
-\infty \leq f^{\prime}\left(x_{0}\right) \leq+\infty
$$

then we say that the function $f$ has a derivative at a point $x_{0}$, and in the case

$$
-\infty<f^{\prime}\left(x_{0}\right)<+\infty-
$$

a finite derivative.

We say that the function $f$ is defined in the broad sense, if

$$
f(x) \in[-\infty ;+\infty]
$$

In other words, function, defined in the broad sense in an $\Delta$ interval, may take finite or infinite values at the points of this interval. For example, the function $f(x)=\infty$ is defined in the broad sense in the $(-\infty ;+\infty)$ interval, but $f(x)=\frac{1}{x-3}$ is not defined at the point $x=3$.

Definition 1.1 (continuity in the broad sense). Let the function $f$ defined at the point $x_{0}$ and in some neighborhood in the broad sense. Then, if there is a limit of the function $f$, when $x \rightarrow x_{0}$ and $\lim _{x \rightarrow x_{0}} f(x)=f\left(x_{0}\right)$, is held 
then the function $f$ is called continuous in the broad sense at this point.

For example, the function

$$
f(x)=\left\{\begin{array}{r}
\frac{1}{(x-5)^{2}}, x \neq 5, \\
+\infty, x=5
\end{array}\right.
$$

is continuous in the broad sense at the point $x=5$, as

$$
\lim _{x \rightarrow 5} f(x)=\lim _{x \rightarrow 5} \frac{1}{(x-5)^{2}}=+\infty=f(5) .
$$

The function, which is continuous in the broad sense in the given interval, may be continuous or continuous in the broad sense at the points of this interval.

\section{A Smooth Curve and a Smooth Function in the Broad Sense}

Let us define a smooth curve in the language of a continuous derivative in the broad sense.

Definition 2.1. The curve $\Gamma \subset R^{3}$ given by parametric equations

$$
x=x(t), y=y(t), z=z(t), t \in[a ; b]
$$

is called smooth, if

$$
x(t), y(t), z(t)-
$$

continuous functions with continuous in the broad sense derivatives in $[a ; b]$ simultaneously not equal to zero.

Definition 2.1. can be used to the curves represented by differentiable functions in the considered interval, as well as to the curves, represented by the functions with infinite derivatives. A special case of the definition 2.1 is the following.

Definition 2.2. The curve

$$
\Gamma: y=f(x), x \in[a ; b]
$$

is called smooth, if the function $f$ has continuous in the broad sense derivative in the interval $[a ; b]$.

For example, according to the definition 2.2 the curve

$$
y=\sqrt[3]{t},-\infty \leq x \leq+\infty,
$$

is smooth on any interval, since the derivative

$$
f^{\prime}(x)=\left\{\begin{array}{c}
\frac{1}{3 \sqrt[3]{x^{2}}}, x \neq 0 \\
+\infty, x=0
\end{array}\right.
$$

is continuous in the broad sense at any endpoint.

Further, suppose that the function

$$
y=f(x), x \in[a ; b]
$$

is given and let its derivative exist in $[a ; b]$. The function

$$
\alpha(x)=\operatorname{arctg} f^{\prime}(x), \quad x \in[a ; b],
$$

Where

$$
f^{\prime}(x) \in[-\infty ;+\infty]
$$

is said to be an angular function for $f$. Here the function $\operatorname{arctg} v$ considered to be defined on the extended set of real numbers $[-\infty ;+\infty]$, with the conditions of

$$
\begin{gathered}
\operatorname{arctg}(+\infty)=\frac{\pi}{2} \text { and } \operatorname{arctg}(-\infty)=-\frac{\pi}{2}, \text { i.e }-\frac{\pi}{2} \leq \\
\alpha(x) \leq \frac{\pi}{2} .
\end{gathered}
$$

Defined this way function arctgv is continuous in $[-\infty ;+\infty]$.

From equation (1) it is seen that the existence of the derivative $f^{\prime}(x)$ is equivalent to the existance of an angular funcition at the point $x$. Similarly, the continuity of the derivative $f^{\prime}(x)$ in the broad sense is equivalent to continuity in the usual sense of the angle funciton $\alpha(\mathrm{x})$.

Indeed, suppose that derivative $f^{\prime}$ at the point $\mathrm{x} 0$ is continuous in the broad sense, i.e.

$$
\lim _{x \rightarrow x_{0}} f^{\prime}(x)=f^{\prime}\left(x_{0}\right),
$$

where $f^{\prime}\left(x_{0}\right) \in[-\infty ;+\infty]$. Then, if $f^{\prime}\left(x_{0}\right) \in$ $(-\infty ;+\infty)$, the angular function $\alpha(\mathrm{x})$ is continuous at the point $x_{0}$ as a composition of two continuous functions. If, for example, $f^{\prime}\left(x_{0}\right)=+\infty$, then

$$
\alpha\left(x_{0}\right)=\operatorname{arctg}(+\infty)=\frac{\pi}{2},
$$

and by the continuity of function $\operatorname{arctg} v$ in $[-\infty ;+\infty]$ we get

$$
\begin{gathered}
\lim _{x \rightarrow x_{0}} \alpha(x)=\lim _{x \rightarrow x_{0}} \operatorname{arctg} f^{\prime}(x) \\
=\operatorname{arctg} \lim _{x \rightarrow x_{0}} f^{\prime}(x)=\operatorname{arctg} f^{\prime}\left(x_{0}\right) \\
=\frac{\pi}{2}=\alpha\left(x_{0}\right)
\end{gathered}
$$

Conversely, suppose the angular function $\alpha$ is continuous at the point $x_{0}$ i.e $\lim _{x \rightarrow x_{0}} \alpha(x)=\alpha\left(x_{0}\right)$, where $\alpha\left(x_{0}\right)=\operatorname{arctg} f^{\prime}\left(x_{0}\right)$. Then,taking into consideration inequalities

$$
-\frac{\pi}{2} \leq \alpha(x) \leq \frac{\pi}{2}, \forall x \in[a ; b]
$$

we have

$$
\begin{aligned}
& \lim _{x \rightarrow x_{0}} f^{\prime}(x)=\lim _{x \rightarrow x_{0}} \operatorname{tg} \alpha(x) \\
& =\operatorname{tg} \lim _{x \rightarrow x_{0}} \alpha(x)=\operatorname{tg} \alpha\left(x_{0}\right) \\
& =\operatorname{tg} \operatorname{arctg} f^{\prime}\left(x_{0}\right)=f^{\prime}\left(x_{0}\right)
\end{aligned}
$$

Here it means that, if, for example 


$$
\alpha\left(x_{0}\right)=\operatorname{arctg} f^{\prime}\left(x_{0}\right)=\frac{\pi}{2}, \text { i.e } f^{\prime}\left(x_{0}\right)=+\infty
$$

Then

$$
\lim _{x \rightarrow x_{0}} \alpha(x)=\frac{\pi}{2}-0
$$

and so

$$
\begin{gathered}
\lim _{x \rightarrow x_{0}} f^{\prime}(x)=\lim _{x \rightarrow x_{0}} \operatorname{tg} \alpha(x) \\
=\lim _{x \rightarrow x_{0}} \sin \alpha(x) . \\
\lim _{x \rightarrow x_{0}} \frac{1}{\cos \alpha(x)}=+\infty=f^{\prime}\left(x_{0}\right)
\end{gathered}
$$

Thus, according to the continuity of the angular function $\alpha$ at the given point, continuity of the derivative $f^{\prime}$ at this point in the broad sense is followed. Therefore Definition 2.2. is equivalent to following definition 2.3 , formulated in terms of the angular funcitons.

Definition 2.3. (in terms of the angular function) The continuous curve

$$
\Gamma: y=f(x), x \in[a ; b],
$$

is called smooth, if $f$ has an angular function, continuous in $[a, b]$. For example, considered above curve

$$
y=\sqrt[3]{x},-\infty<x<+\infty
$$

is smooth according to the definition 2.3.,too. Indeed, for

$$
f(x)=\sqrt[3]{x}
$$

which is continuous at any point, angular function

$$
\alpha(x)=\left\{\begin{array}{l}
\operatorname{arctg} \frac{1}{\sqrt[3]{x^{2}}}, x \neq 0 \\
\frac{\pi}{2}, x=0
\end{array}\right.
$$

is defined.

For the function

$$
f(x)= \begin{cases}x^{2} \sin \frac{1}{x}, & x \neq 0, \\ 0, & x=0\end{cases}
$$

the angular function equal to

$$
\alpha(x)= \begin{cases}\operatorname{arctg}\left(2 x \sin \frac{1}{x}-\cos \frac{1}{x}\right), & x \neq 0, \\ 0, & x=0,\end{cases}
$$

is defined, but it is not continuous at the point $x=0$ ( the function $\cos \frac{1}{x}$ has no limit at $x \rightarrow 0$ ), so the curve, given by the function $f$, is not smooth in sense of the definition 2.3 in the interval, which has the point $x=0$.

Let $A(x ; f(x))$ to be some point of continuous curve $\Gamma$ : $y=f(x), x \in[a, b]$. We take the point $(x+h) \in(a, b)$ and choose the direction of the line $S$, which passes through the point $A(x ; f(x))$ and $B(x+h ; f(x+h))$, so that the angle $\beta$ between the positive direction of the $\mathrm{O} x$ axis and direction of the line $\mathrm{S}$ would be sharp: $-\frac{\pi}{2}<\beta<\frac{\pi}{2}$. We call directed line $\mathrm{S}$, which is obtained in such a way, the secant, and $\beta=\beta(x ; h)$ its angle of inclination.

Definition 2.4. If there is a limit $\lim _{h \rightarrow 0} \beta(x, h)$, then directed line $\mathrm{T}$ (limiting position of directional secant), passing through the point $A(x, f(x))$ with the angle of inclination $\alpha(x)=\lim _{h \rightarrow 0} \beta(x, h)$, is called tangent to the curve at this point.

Note that the angle of inclination of the secant is in the range $\left(-\frac{\pi}{2} ; \frac{\pi}{2}\right)$, while the slope of the tangent can take values from the interval $\left[-\frac{\pi}{2} ; \frac{\pi}{2}\right]$.

Thus,

$$
\alpha(x)=\operatorname{arctg} \lim _{h \rightarrow 0} \beta(x, h)=\operatorname{arctg} f^{\prime}(x)
$$

is the slope of the tangent to the curve at the point with abscissa $x$. Consequently, the existence of the derivative of the function $f$ at the point $\mathrm{x}$ implies the existence of a tangent to the curve at the point with abscissa $x$. However, the fact that a tangent exists at the point of the curve with abscissa $x$ is possible, but the function, which defines this curve is not differentiable at the point $x$.

Further, let the angular function $\alpha=\alpha(x)$ to be defined for the function $\mathrm{f}$ at the point $\mathrm{x}$ and some neighborhood. Then the tangent at the point $(x, f(x))$ of the graph of the function $f$ is continuous at the point $x$, if the angular function $\alpha$ is continuous at this point. We say that the tangent of the graph of the function $f$ is continuous in the interval $\Delta$, if it is continuous at each point of the $\Delta$.

Definition 2.5. (in terms of the tangent) The continuous curve $\Gamma: y=f(x), x \in[a ; b]$ is called smooth, if there is a continuous tangent of the curve $\Gamma$ in the interval $[a ; b]$.

Let us introduce the concept of the smooth function in the broad sense.

Definition 2.6. Function $f$ is called smooth in the broad sense in the interval $[a ; b]$, if it has continuous in the broad sense derivative in this interval.

Definition 2.7. Continuous curve $\Gamma: y=f(x), x \in[a, b]$ is 
called smooth, if the function $f$ in the interval $[a ; b]$ is smooth in the broad sense.

For the curve, given parametrically, definition 2.7. can be summarized as follows.

Definition 2.8. Continuous curve $\Gamma \subset R^{3}$, given parametrically by equations $x=x(t), \quad y=y(t)$, $z=z(t), t \in[a ; b]$, is called smooth in the interval $[a ; b]$, if $x(t), y(t), z(t)$ are smooth functions in the broad sense, which have simultaneously not equal to zero derivatives in the interval $[a ; b]$.
Some provisions of the questions, discussed in this article are presented in [2], in style of the requirement of the curriculum of technical universities.

\section{References}

[1] S.M. Nikolskyi. Course of Mathematical Analysis, Volume 1, "Science", 1983.

[2] E.Zh. Aydos. Higher mathematics-2, "Bastau", Almaty, 2010. 